\title{
Preparation and Characterization of Artificial Spider Silk Produced through Microchannel Techniques
}

\author{
Abdalla S*, Obaid A, Al-Marzouki F and Bahabri F \\ Department of Physics, Faculty of Science, King Abdulaziz University, Jeddah, P.O. Box 80203, Jeddah 21589, Saudi Arabia
}

\begin{abstract}
Spider silk (SS) is naturally tough; however, it turns soft when wet by water. Spiders produce high-quality silk threads by adjusting the molecular assemblage of SS-proteins and the arrangements structure of threads and recombinant spider dragline silk (RSDS). The general wet spinning techniques for producing recombinant spidroins results in uncorrected explanation to the natural spinning technique. In this study, we use tailored-SS with relative low molecular weight of $47 \mathrm{kD}$ to produce a water-soluble RSDS protein. We built a microfluidic ship and used it to spun SS using aqueous solutions-micro-technique (wet spinning). This was done in order to mimic the spider-spinning processes using a steady post-spin drawing process. We succeeded to produce assemblies of spidroins with fibril structure. Then, compact constituting of micro-threads followed these wet spinning processes. Wet spinning was followed by improving the orientation, crystalline structure, and fibril melting of the hierarchical structure. The initial mechanical characterization (tensile strength) of the RSDSs attained about $510 \mathrm{MPa}$ with respective extension $44 \%$.
\end{abstract}

Keywords: Artificial spider silk; Aqueous solution technique; Funnel microfluidic channel; Mechanical properties; Structural defects of spidroins

\section{Introduction}

Spider Silks (SSs) can be comparable to steel at a trivial quantity of weight, and even they can be more flexible than rubber and tougher. SSs are nontoxic and have some medical applications such as them to be likely behaved to stimulate human immune cells [1]. Certain types of SSs prevents bacteria and fungi, allowing them to be ideal biomaterials for microsurgery and, even medical device applications [2,3]. Gatesy et al. [4] are of the first to point out about the importance of SSs. However, researchers found huge slow down to exploit these natural wonderful biomaterials, perhaps due to the inhabitations concerned in deep well-studying and recognizing spider silk genes. However, scientists, nowadays, restart to carry out an important progress in this direction with the most important-ever study of spider silk genes [1,47]. SSs is an interesting bioorganic compound and it has very low mass with stiffness compared to the stress of steel. However, unfortunately, industrial production of these SSs is now a challenge as spiders produce very limited quantities of their SSs. As a direct consequence, producing industrial rate should implicate the direct usage of human-made silk compounds with human spinning technologies. Researchers starts to mimic spiders and turn to biomimetic-spinning technologies [2,8-12]. Up to date, they did not reach to their aim. This is because obtaining water-soluble spider silk proteins is very difficult. Therefore, one must think about stronger solvents that can solve this problem. In nature, before giving silk RSDSs, spider keep this protein (silk) in an aqueous solution in their silk glands; this protein is termed spidroin [2]. Several researchers [2,8-12] have found remarkable $\mathrm{pH}$ variation in these glands. They [2,8-12] marked that these controlled variations stimulate certain portions of the SSs-protein, which initiates the production of RSDS in certain well localized (and recognized) point. Mimicking this known knowledge, one can produce industrial quantities of SSs. Interestingly; these authors [8-12] obtained a water-soluble protein, which exactly mimic nature. This is important because one can now keep the spider protein soluble at very high amounts, which enables spider dragline silk RSDS formation. RSDS keep the same characteristics as the natural SSs, i.e. it has remarkable mechanical characteristics with high stiffness, high degree of strain and extraordinary flexibility. In this regard, RSDS contains several spidroins, which resemble organic polymer having a special hierarchical structure; one can compare the flexibility of RSDS to rubber and its stress compared to steel $[13,14]$. Cameron et al. [15] have reported that RSDS contains a huge number of tinny crystallites (of alanine-rich) surrounded by amorphous (glycine-rich) domains having flexible rubber-like chains. In addition, several authors [16,17] reported that $\mathrm{H}_{2}$-bonds connect the tinny crystallites to the amorphous domains that form RSDS. After this analysis, one can see that the extraordinary mechanical properties of RSDS is due to intra-molecular unfolding behavior of $\mathrm{H}_{2}$-bonds (flexibility) and the high stiffness is due to the presence of nano crystallites.

Moreover, several authors have shown that spinning processes drastically affect the formation of RSDS [18-20]. If one takes the same hierarchical structure with same similar sequences of amino acid, one will obtain RSDS-protein. Therefore, in order to get hand-made SSs (HSSs), one must substitute several recombinant spidroins as feedstocks. Tokareva et al. [21] have found that HSSs with large protein molecules contain alanine-rich and glycine/proline-rich motifs. These HSSs have low molecular weight and have relatively weak mechanical properties. In addition, the HSSs with higher molecular weight cannot solute in aqueous solutions at high concentrations which gets one back to the spinning mechanisms. In fact, silkworm and spider both use microfluidic technique and we will use this technique to spin HSSs.

In particular, the longitudinal flow through long microfluidic channel (MFC) is substantial factor to assemble the recombinant spider proteins $[22,23]$. The anatomy of silk gland in the major ampullate and

${ }^{*}$ Corresponding author: Soliman Abdalla, Department of Physics, Faculty of Science, King Abdulaziz University, Jeddah, P.O. Box 80203, Jeddah 21589, Saudi Arabia, Tel: +966-58-234-3822; E-mail: smabdullah@kau.edu.sa

Received October 25, 2017; Accepted October 30, 2017; Published October 31, 2017

Citation: Abdalla S, Obaid A, Al-Marzouki F, Bahabri F (2017) Preparation and Characterization of Artificial Spider Silk Produced through Microchannel Techniques. J Material Sci Eng 6: 383. doi: 10.4172/2169-0022.1000383

Copyright: ( 2017 Abdalla S, et al. This is an open-access article distributed under the terms of the Creative Commons Attribution License, which permits unrestricted use, distribution, and reproduction in any medium, provided the original author and source are credited. 
in silkworm gland are the same [24]. This anatomy shows that they are both have funnel-structure where radius goes finer with elongation. Glands in both insects function with the same mechanism: They form crystalline liquid by varying the ionic gradients that controls the production of protein [25]. We started by designing a funnel-shape microfluidic channel following the same technique as we described in reference [26]. Figure 1a shows the details of this MFC.

We started by designing a funnel-shape microfluidic channel following a modified technique as we described in reference [26]. Figure la shows the details of this MFC; it is $2.065 \mathrm{~mm}$ initial diameter, $0.265 \mathrm{~mm}$ final diameter and $21.5 \mathrm{~mm}$ length. We expressed engineered Escherichia coli to stand for feedstock for spinning dope, where the recombinant major ampullate spidroin of the spider Nephila clavipes has a relatively weak molecular weight of $47 \mathrm{kDa}$. This poor molecular weight enables the facile use of spidroins in water. We used coagulation ethanol-bath to cover the microfluidic channel that leads to wetspinning processes. Figure $1 \mathrm{~b}$ illustrates the wet spinning technique. In Figure 1c, we modified the setup in such a way that the obtained file passes and dried in air certain period before rolling it. The drying rate is an important factor. One should note that the continuous withdrawal process of the RSDS and get it out from the alcohol bath drying in air improves its mechanical quality. As the drying time increases, the obtained RSDS tends to have more homogeneous and more compact properties Figure 2

\section{Methods}

\section{Preparation of recombinant dragline silk proteins and spinning dope}

The output recombinant protein is water-soluble and to keep it stable and soluble at high concentration, the aqueous silk protein (spidroin) aqueous (AS) was dialyzed in $150 \mathrm{mM} \mathrm{NaCl} \mathrm{AS.} \mathrm{Then,} \mathrm{the}$ dilute AS was concentrated through robust air stream flow as it was explained by Peng et al. [27]. Sodium chloride crystals precipitated from the AS with minute concentrations in such a way that the total concentration of the protein and sodium chloride remains about of 42 wt. \%. The rest of sodium chloride saturated in the dope as nearly all
$\mathrm{H}_{2} \mathrm{O}$ content was water evaporated. We used an oven at $105^{\circ} \mathrm{C}$, for two hours, to dry drop of the dope on a glass bar; following by weighting the bar before and after drying. This means that the weight percent of the resting solid with respect to the weight of the drop before drying is the mass of sodium chloride and protein. We carried out cycles of measurements of at least (4) measurements taken subsequently.

Dissolving about $35.7 \mathrm{~g}(\mathrm{mn})$ in $100 \mathrm{~mL} \mathrm{H}_{2} \mathrm{O}$, one can obtained the solubility $\left(\mathrm{S}_{\mathrm{NaCl}}\right)$ of $\mathrm{NaCl}$ as follows: $\mathrm{S}_{\mathrm{NaCl}}=$ [the protein weight $(\mathrm{mp})$ $+\mathrm{NaCl}$ weieght $(\mathrm{mn})] /[($ the protein weight $+\mathrm{NaCl}$ weieght +100$)]$ should equal 42 percent. Therefore, the concentrations of protein and sodium chloride in the spinning dope are 20.7 wt. percent and 21.3 percent, respectively. In the as-prepared RSDSs, we calculated the wt. percent of sodium chloride as to be $50 \%$.

\section{Spinning apparatus and RSDS formation}

We designed an artisanal microfluidic channel to be part of a spinning apparatus attached with a set up to produce mechanical forces that creates shearing gradients. We mimic the function of silk gland $[28,29]$ in such a way that the diameter of microfluidic channel vary from initial input point $\mathrm{D}_{\text {in }}=2.065 \mathrm{~mm}$ down to $\mathrm{D}_{\text {out }}=0.265 \mathrm{~mm}$, with a total length $21.5 \mathrm{~mm}$. Figure 1a illustrates these details. In Table 1, we compare the dimension details of our microfluidic channel with the duct of Nephila edulis and B. mori $[7,24]$. We start the spinning processes by injecting the dope into the microfluidic channel, then, pushed it into the alcoholic bath. The rate of obtaining the as-prepared RSDSs was about three $\mathrm{cm}$ per second and the rate of obtaining the dried RSDSs was the same value, in both cases ethyl alcohol keep the coagulation effects.

\section{Results and Discussions}

Figure $2 \mathrm{a}$ shows the wet as-prepared RSDS. Figure $2 \mathrm{~b}$ illustrates the RSDS where we have subjected it when it was inside the microfluidic channel to mechanical stresses, before embedding the RSDS into alcohol bath. The obtained results are not far from those obtained by Jin et al. [30]. One notices that the RSDS contains some aggregated spidroin bundles through the long-axis of the RSDS. Jin et al. [30] and Xia et al. [31] have reported that both recombinant dragline and natural

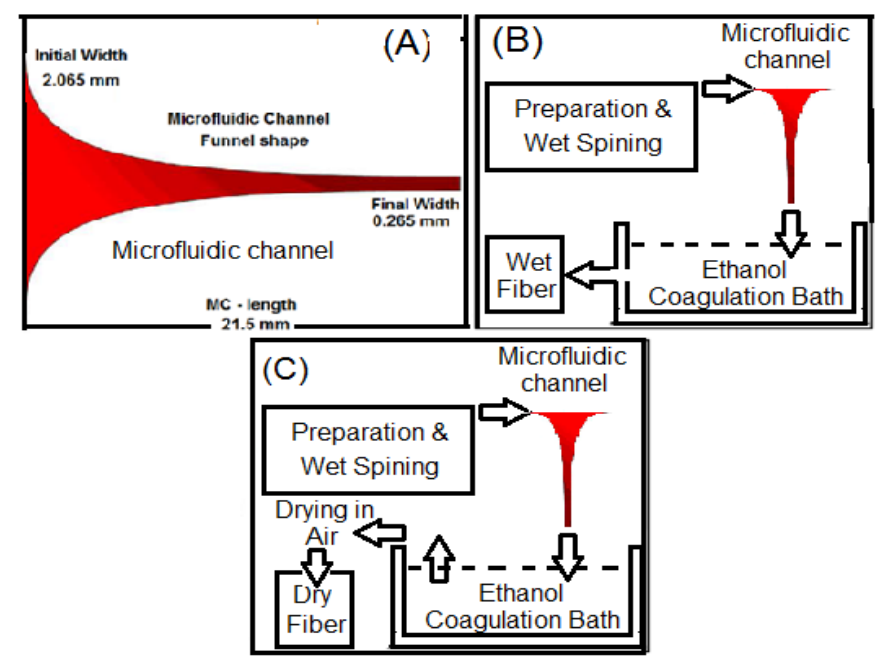

Figure 1: Symbolic illustrations of the present microfluidic technique in order to produce silk fiber of accepted quality: (a) Dimensions of the proposed microchannel (MC) used as spinning-duct. One can control contracting spinning ducts in order to be more compact and more homogenous using lateral and longitude stresses. We use the dimensions of the silkworm silk gland as shown in Table 1. (B) Wet-spinning micro fluidic technique to produce as-prepared fibers $F_{0}$ and $F_{3}$. $(C)$ Wet-spinning micro fluidic technique to produce the treated fibers $F_{1}, F_{2}, F_{4}$ and $F_{5}$. The diameter of the $M C$ lowers in an exponential function to from a funnel. 


\begin{tabular}{|c|c|c|}
\hline Spinning duct & Initial Width $(\mathbf{m m})$ & Final Width $(\mathbf{m m})$ \\
\hline Nephila edulis MA-spider & 0.260 & 0.020 \\
\hline B. mori (silkworm) & 0.413 & 0.053 \\
\hline Bio-microfluidic channel & 2.065 & 21.5 \\
\hline
\end{tabular}

Table 1: Parameters of spinning ducts.

spider dragline silk have the fibrillary structure. Close examination on data of Figure 2a reveal the presence of granules on the RSDS surface, in addition to some important cracks. These irregular structures are in harmony with the presence of some net-pores and disordered voids in the cross section Figure $2 \mathrm{~b}$. On the contradictory, Figure $2 \mathrm{c}$ and $2 \mathrm{~d}$ illustrate homogenous RSDS. The withdrawal process in air applies certain stresses that lead to certain homogenization in the structures.

We then imbedded the dried samples in $\mathrm{H}_{2} \mathrm{O}$ for about 60 s to more solving sodium chloride. As seen from Figure 2e, the RSDS of dried samples shows more compact fibril structure. This RSDS showed structure stability in $\mathrm{H}_{2} \mathrm{O}$ and air. We carried out another treatment on the dried samples: stretching thrice at a rate $0.9 \mathrm{~mm}(\mathrm{sec})^{-1}$ in ethyl alcohol (70 vol. \%). This stretching treatment resulted in more homogenization with more orientation.

\section{Spider dragline silk morphology with different treatments}

Figure 2e shows no net variations in morphology of dried samples. We immersed these samples in 70 vol. percentage ethyl alcohol aqueous solution for about five seconds to remove a part of sodium chloride the water-soluble as-prepared RSDSs become water insoluble RSDSs. Figure $2 \mathrm{~g}$ illustrates fibril structure however; there are net cracks all over the surface RSDS. When we carried out mores stretching, we get more homogenous RSDS as one can note in Figure $2 \mathrm{~h}$.

In order to get the best quality of RSDS, we tried several possibilities and treated the as prepared RSDS ( $\left.\mathrm{F}_{0}\right)$ by five steps as following: (1) we embedded the as prepared RSDS in ethyl alcohol AS (70 vol. \%) for five seconds. This process removes small quantity of sodium chloride and yield the first step of our RSDS-treatment process RSDS noted as $\left(\mathrm{F}_{1}\right)$. This RSDS is very hard to dissolve in water and it is steady in both water and air, whereas, on the contrary $F_{0}$ was soluble in water $\left(F_{2}\right)$. (2) We embedded $F_{1}$ in water for a period of 60 seconds in order to get rid of more sodium chloride $\left(\mathrm{F}_{3}\right)$ (Figure 2e).

(3) One carried out the third treatment by a gentle but strong stretch of the as prepared RSDS through stretching thrice with rate about $1 \mathrm{~mm}$ per seconds (in 70 vol. percentage ethyl alcohol AS) $\left(\mathrm{F}_{4}\right)$. (4) The fourth treatment is nearly the same as the third one but here we dried the RSDS continuously in air. (5) Finally, the last treatment, which leads to the best results, occurred when we withdraw the RSDS $\mathrm{F}_{4}$ in air for longer period leading to $\mathrm{F}_{5}$. Thus, we have two types of treatment; one in ethyl alcohol and the second is in air. Another type of treatment is gentle stretching of the RSDS. Table 2 summarizes the five different treatment steps.

In order to get the best quality of RSDS, we tried several possibilities and treated the as prepared RSDS $\left(\mathrm{F}_{0}\right)$ by five steps as following: (1) we embedded the as prepared RSDS in ethyl alcohol AS (70 vol. \%) for five seconds. This process removes small quantity of sodium chloride and yield the first step of our RSDS-treatment process RSDS noted as $\left(\mathrm{F}_{1}\right)$. This RSDS is very hard to dissolve in water and it is steady in both water and air, whereas, on the contrary $\mathrm{F}_{0}$ was soluble in water $\left(\mathrm{F}_{2}\right)$. (2) We embedded $F_{1}$ in water for a period of 60 seconds in order to get rid of more sodium chloride $\left(\mathrm{F}_{3}\right)$ (Figure 2e). (3) One carried out the third treatment by a gentle but strong stretch of the as prepared RSDS
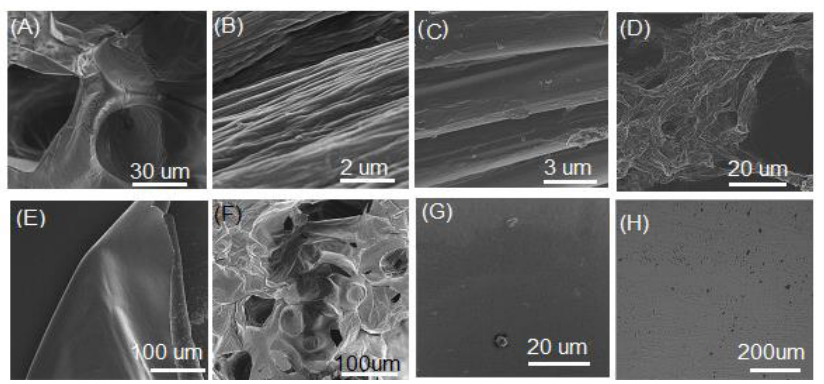

Figure 2: Scanning Electron Microscope images of recombinant spider silks: (A) Surface of as prepared wet RSDS $F_{0}$, (B) cross-sectional image of $F_{0}$, (C) Surface of RSDS after stretching in alcohol $F_{1},(D)$ cross-sectional image of $F_{1}$, (e) Surface of stretched RSDSs in methyl alcohol $F_{2}$, (F) Dried samples- $F_{3}$, (G) Stretched RSDSs in alcohol $F_{4}(H)$ Stretched RSDSs in alcohol and dried in air $F_{5}$.

through stretching thrice with rate about $1 \mathrm{~mm}$ per seconds (in 70 vol. percentage ethyl alcohol AS) $\left(\mathrm{F}_{4}\right)$. (4) The fourth treatment is nearly the same as the third one but here we dried the RSDS.

\section{Structure of recombinant-spider dragline silks}

As one sees in Figure 3, the wide-angle X-rays diffraction patterns and corresponding one-dimensional diffracto-grams of recombinant spider dragline silk RSDSs confirm the above-mentioned data. Comparing data in Figure 3a-3g, 2D wide-angle X-rays diffraction patterns of the as prepared files demonstrates a net diffusion halo corresponding to no peak in the 1D-pattern. One can explain this behavior on the fact that the as-prepared file contains large numbers of disordered tinny crystallites that results in high ability to dissolve in $\mathrm{H}_{2} \mathrm{O}$. Figure $3 \mathrm{~b}$ shows that the diffusion halo in Figure $3 \mathrm{a}$ turns into two dependent diffusion rings, and the corresponding one dimension diffracto-gram illustrated in Figure $3 \mathrm{~g}$ shows two obvious diffraction peaks at the location of the (120-reflection plane with $\mathrm{d}=4.36$ Angstroms) and the (211-reflection plane with $d=3.73$ Angstroms). These data are in harmony with those obtained for spider dragline silk by Sampath et al. [32]. One can attribute the improvement of RSDSs properties to the homogeneity of crystal structure occurred by drying treatment and longitudinal mechanical stresses. Moreover, the present experimental data demonstrate that more wetting of RSDS stimulate the production of more homogenous tinny crystals with fine structure. This fact can be seen from the results illustrated in Figure $3 \mathrm{c}$ and $3 \mathrm{~g}$ where net peaks at (120) and (211), planes are shown. Water molecules enhance the motion of polymeric-chains through diffusion into the amorphous regions and stimulating the intermolecular interactions when sodium chloride is dissolved. Figure $3 \mathrm{e}$ shows that there is net diffraction arcs at the plane located at 200 and another at 120. This shows that crystalline domain was directed longitudinal through the RSDS at the period of withdrawal (drying process) in air. This crystalline formation is confirmed from data on Figure $3 \mathrm{~d}$ and $3 \mathrm{f}$ where crystalline domains were directed longitudinally through the RSDS after the mechanical stretching treatment. In addition, one can notice the presence of semi-crystalline structure with nano crystallites inlayed through the amorphous regions [32]. 


\begin{tabular}{|c|c|c|}
\hline RSDS No & Description & Experimental \\
\hline $\mathrm{F}_{0}$ & As prepared RSDS. $F_{1}$ is soluble in water and unstable in air. Figure $2 A$ : surface of $F_{0}$ and $2 B$ : cross-section of $F_{0}$. & $\begin{array}{l}\text { Figure } 2 A \text { : surface of } F_{0} \text { and } \\
\text { 2B: cross-section of } F_{0} \text {. }\end{array}$ \\
\hline $\mathrm{F}_{1}$ & $\begin{array}{l}\text { We embedded the as prepared RSDS in ethyl alcohol AS }(70 \text { vol.\%) and stretched for five seconds. This process } \\
\text { removes small quantity of sodium chloride } F_{1} \text { is very hard to dissolve in water and it is stable in both water and air. }\end{array}$ & $\begin{array}{l}\text { Figure } 2 C \text { : surface of } F_{1} \text { and } \\
2 D \text { : cross-section of } F_{1} \text {. }\end{array}$ \\
\hline $\mathrm{F}_{2}$ & $\begin{array}{l}\text { We get } F_{2} \text { by the same technique as } F_{0} \text { but here we dried the RSDS in air for } 60 \text { seconds. } F_{2} \text { is very hard to dissolve in } \\
\text { water and it is stable in both water and air. It is more homogenous than } F_{1} \text {. }\end{array}$ & Figure 2E: surface of $F_{2}$ \\
\hline $\mathrm{F}_{3}$ & $\begin{array}{l}\text { One gets } F_{3} \text { by the same technique as } F_{1} \text { but here we dried the RSDS in air for } 60 \text { seconds. } F_{2} \text { is very hard to dissolve in } \\
\text { water and it is stable in both water and air. It is more homogenous than } F_{1} \text {. }\end{array}$ & Figure 2F: surface of $F_{3}$ \\
\hline $\mathrm{F}_{4}$ & $\begin{array}{l}\text { One gets } \mathrm{F}_{4} \text { by carrying out the same treatment by a gentle but strong stretch of the as prepared RSDS through } \\
\text { stretching thrice with rate about } 1 \mathrm{~mm} \text { per seconds (in } 70 \text { vol. percent) in ethyl alcohol }\end{array}$ & Figure 2G: surface of $F_{4}$ \\
\hline $\mathrm{F}_{5}$ & $\begin{array}{l}\text { One gets } F_{5} \text { carrying out the same treatment by a gentle but strong stretch of the as prepared RSDS through stretching } \\
\text { thrice with rate about } 1 \mathrm{~mm} \text { per seconds (in } 70 \text { vol. percent) in air }\end{array}$ & Figure $2 \mathrm{H}$ : surface of $\mathrm{F}_{5}$ \\
\hline
\end{tabular}

Table 2: Five different treatment steps to produce spider silk.

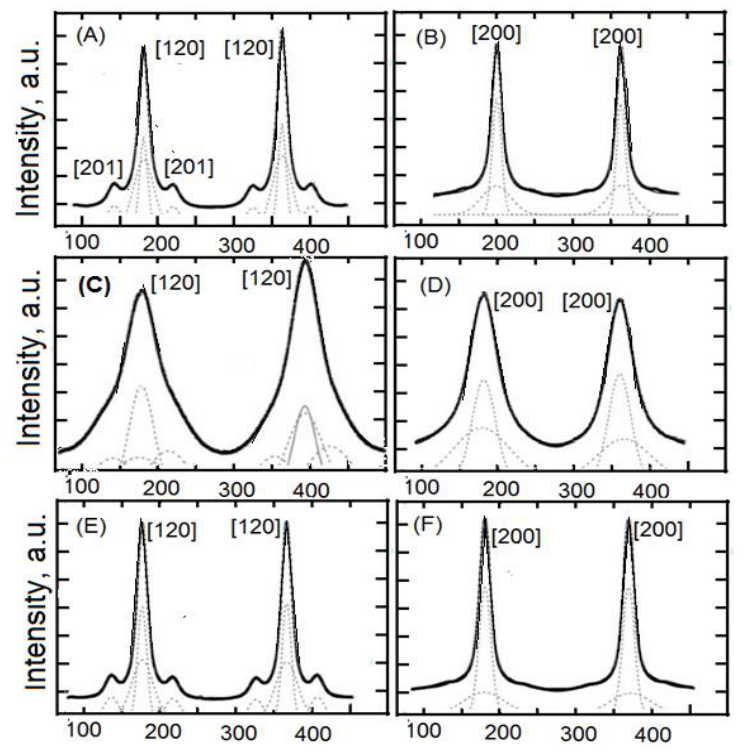

Figure 3: $D$-spacing pattern and structures of RSDS fibers. $(A) F_{0},(B) F_{1},(C) F_{2},(D) F_{3},(E) F_{4},(F) F_{5}$, and $(G)$ corresponding one dimension diffraction pattern.

\section{Mechanical properties of recombinant-spider dragline silks}

We illustrate Young's curves for both as-prepared and dried RSDSs in Figure $4 \mathrm{a}$ and $4 \mathrm{~b}$. These figures show the stresses forces as a function of the corresponding strains and these mechanical properties can be compared to recombinant spider silk found in literature. In Table 3, we report mechanical values of natural spider dragline silk compared to the data of the present work. Our average values of critical strengths and strains (breaking points) for the RSDSs $\mathrm{F}_{4}$ and $\mathrm{F}_{5}$ reported as the following: $62.3 \mathrm{MPa} \pm 17.2 \mathrm{MPa}$ and $3.5 \% \pm 1.2 \%$, respectively.

We found optimum values: Critical cutoff strength is up to 510 $\mathrm{MPa}$ with corresponding strain was up to $44 \%$ (Figure $4 \mathrm{~b}$ ).

Concerning $\mathrm{F}_{5}$, the mechanical properties (Table 3) demonstrate, regrettably, important variability. One can attribute this variability to the inhomogeneous distribution of sodium chloride through RSDS. In addition, the variations of spidroin molecular structure can affect these mechanical properties.

Heidebrecht et al. [22] have found that starting with protein with molecular weight $120 \mathrm{kD}$ produced excellent flexible RSDSs having cutoff strength of $383 \mathrm{MPa}$ with corresponding strain of $600 \%$ [22]. When one compare the mechanical data $\mathrm{F}_{5}$ of molecular weight $47 \mathrm{kD}$ with the results obtained by Heidebrecht et al. [22], one can find relatively good output because the present technique uses economic and nontoxic ASs.

Although much smaller spidroins were used compared to the building blocks in this study, WS-PSD-3x shows comparable mechanical properties to the reported recombinant spider dragline silk. However, our spinning dope is an aqueous solution and the spinning process is thus economic and non-toxic.

\section{Conclusions}

We demonstrated that the wet spinning with microfluidic techniques could produce relatively good quality of recombinant spider dragline silk protein $(47 \mathrm{kDa})$. When fiber is dried in air, F1, it forms compact sort of protein. Additional stretching forces let more homogenization in the bulk of the fiber. This allows the fiber to get $510 \mathrm{MPa}$ stresses and $44 \%$ strains. The proposed technique is economic and nontoxic at all. We predict that if one adds amino-terminal or/ and carboxy-terminal, this would lead to better spinning recombinant proteins with poor salt concentration and higher molecular weight.

\section{Acknowledgements}

This project was supported by King Abdulaziz City of Science and Technology, general direction of research grants - Project No. (ARP-35-157), the author also, acknowledge with thanks the Deanship of Scientific Research, King Abdulaziz University for technical support. 
Citation: Abdalla S, Obaid A, Al-Marzouki F, Bahabri F (2017) Preparation and Characterization of Artificial Spider Silk Produced through Microchannel Techniques. J Material Sci Eng 6: 383. doi: 10.4172/2169-0022.1000383

Page 5 of 6

\begin{tabular}{|c|c|c|c|c|c|c|}
\hline Protein origin & Molecular weight (kDa) & Solvent/coagulation bath & Breaking Strain (\%) & Breaking Stress (MPa) & Modulus (GPa) & Toughness (MJm $\left.\mathbf{~}^{-3}\right)$ \\
\hline$F_{4}$ & 47 & Water/ethanol & $3.5 \pm 1.2$ & $62.3 \pm 17.2$ & $4.0 \pm 2.8$ & $1.6 \pm 0.95$ \\
\hline$F_{5}$ & 47 & Water/ethanol & $18.3 \pm 12.8$ & $286.2 \pm 137.7$ & $8.4 \pm 4.3$ \\
\hline Natural Silk & 2250 & water & $24 \pm 8$ & $1183 \pm 334$ \\
\hline
\end{tabular}

Table 3: Prepared silk characteristics compare to natural spider silk RSDSs.
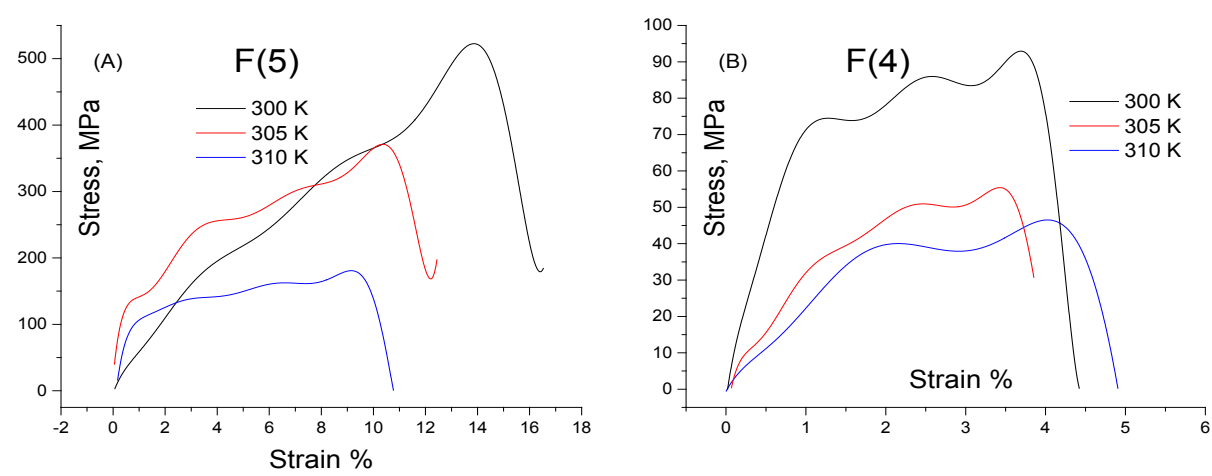

Figure 4: Mechanical characteristics of obtained spider silk fibers (RSDS). Young's curves (strain-stress curves) of fiber $F_{4}$ are illustrated in (A) and those of fiber $F_{5}$ are illustrated in (B). Different curves in each image depict another cycle of measurements, the variability of the successive data is attributed to variation of sodium chloride concentrations and it is explained in text.

\section{References}

1. Paul LB, Nicholas FL, Sandra MCG, David NN, Eun JK, et al. (2017) The Nephila clavipes genome highlights the diversity of spider silk genes and their complex expression. Nature Genetics.

2. Wu S, Johansson J, Hovatta O, Rising A (2015) Efficient passage of human pluripotent stem cells on spider silk matrices under xeno-free conditions. Cell Mol Life Sci. Oct 1.

3. Abdalla S, Obaid A, Al-Marzouki F, Bahabri F (2017) Tailored Spider Silk: The Future Solution to Biomaterials, Editorial opinion. J Material Sci Eng 6: 5

4. Gatesy J, Hayashi C, Motriuk D, Woods J, Lewis R (2001) Extreme diversity, conservation, and convergence of spider silk fibroin sequences. Science 291 : 2603-2605.

5. Hayashi CY, Lewis RV (2000) Molecular architecture and evolution of a modular spider silk protein gene. Science 287: 1477-1479.

6. Sanggaard KW, Jesper SB, Xiaodong F, Jinjie D, Thomas FD, et al. (2014) Spider genomes provide insight into composition and evolution of venom and silk. Nat. Commun 5: 3765.

7. Kuwana Y, Sezutsu H, Nakajima K, Tamada Y, Kojima K (2014) Hightoughness silk produced by a transgenic silkworm expressing spider (Araneus ventricosus) dragline silk protein.

8. Andersson M, Jia Q, Abella A, Xiau-Yeen Lee, Landreh M, et al. (2017) Biomimetic spinning of artificial spider silk from a chimeric mini-spidroin. Nature Chemical Biology.

9. Rising A, Johansson J (2015) Toward spinning artificial spider silk. Nat Chem Biol 11: 309-15.

10. Andersson M, Chen G, Otikovs M, Landreh M, Nordling K, et al. (2014) Carbonic Anhydrase Generates CO2 and H+ That Drive Spider Silk Formation Via Opposite Effects on the Terminal Domains. PLoS Biol 5: 12: e1001921.

11. Kronqvist N, Otikovs M, Chmyrov V, Chen G, Andersson M, et al. (2014) Sequential $\mathrm{pH}$-driven dimerization and stabilization of the $\mathrm{N}$-terminal domain enables rapid spider silk formation Nat Comm 10: 3254

12. Yuchao Wu, Darshil US, Chenyan Liu, Ziyi Yu, Ji Liu, et al. (2017) Bioinspired supramolecular SDSs drawn from a multiphase self-assembled hydrogel. Proceedings of the National Academy of Sciences.

13. Jinrong Wu, Li-Heng Cai, David AW (2017) Tough Self-Healing Elastomers by Molecular Enforced Integration of Covalent and Reversible Networks. Advanced Materials.
14. Li J, Celiz AD, Yang J, Yang Q, Wamala I, et al. (2017) Tough adhesives for diverse wet surfaces. Science 357: 378 .

15. Cameron PB, Federico R, Enrico Traversa d, Silvia L (2011) Spider silk as a load bearing biomaterial: tailoring mechanical properties via structural modifications 3: 870-876.

16. Yan ZHANG, Yu-Yue CHEN, Ling LIN, Pi-bo MA (2014) Nanostructure Characterization of Beta-Sheet Crystals N Silk under various temperatures Thermal Science 1459-1461.

17. Du N, Yang Z, Liu XY, Li Y, Xu HY (2011) Structural origin of the strain hardening of spider silk. Adv Funct Mater 21: 772-778.

18. Vollrath F, Knight DP (2001) Liquid crystalline spinning of spider silk. Nature 410: 541-548.

19. James Sparkes, Chris Holland (2017) Analysis of the pressure requirements for silk spinning reveals a pultrusion dominated process, Nature Communications 8: 594.

20. Celia Henry Arnaud (2017) Process mimics spider silk spinning- Combining parts of silk proteins from various spider species improves production of artificial silk 3: 5 .

21. Olena Tokareva, Valquíria A Michalczechen-Lacerda, Elíbio L Rech, David L Kaplan (2013) Recombinant DNA production of spider silk proteins, Microb Biotechnol 6: 651-663.

22. Heidebrecht A, Eisoldt L, Diehl J, Schmidt A, Geffers M, et al. (2015) Biomimetic fibers made of recombinant spidroins with the same toughness as natural spider silk. Adv Mater 27: 2189-2194.

23. 23] Nilebäck L, Chouhan D, Jansson R, Widhe M, Mandal BB, et al. (2017) Silk-Silk Interactions between Silkworm Fibroin and Recombinant Spider Silk Fusion Proteins Enable the Construction of Bioactive Materials., ACS Appl Mater Interfaces 9: 31634-31644.

24. Wang X, Li Y, Liu Q, Xia Q, Zhao P,(2017) Proteome profile of spinneret from the silkworm, Bombyx mori, Proteomics.

25. Greta Gronau, Zhao Qin, Markus JBuehler (2013) Effect of sodium chloride on the structure and stability of spider silk s $\mathrm{N}$-terminal protein domain. Biomater 1: 276.

26. S Abdalla, FM Al-Marzouki, Ahmed A Al-Ghamdi (2017) Field Effect Transisto using Carbon nanotubes and DNA as Electrical Gate, Braz. J Phys 47: 34-41.

27. Peng Q, Shao H, Hu X, Zhang $Y$ (2017) Microfluidic Dry-spinning and Characterization of Regenerated Silk Fibroin Fibers. J Vis. 
Citation: Abdalla S, Obaid A, Al-Marzouki F, Bahabri F (2017) Preparation and Characterization of Artificial Spider Silk Produced through Microchannel Techniques. J Material Sci Eng 6: 383. doi: 10.4172/2169-0022.1000383

28. Blackledge TA (2013) Spider Silk: Molecular Structure and Function in Webs In: Nentwig W. (eds) Spider Ecophysiology. Springer, Berlin, Heidelberg.

29. Davies GJG, Knight DP, Vollrath F (2013) Chitin in the Silk Gland Ducts of the Spider Nephila edulis and the Silkworm Bombyx mori.

30. Jin HJ, Kaplan DL (2003) Mechanism of silk processing in insects and spiders. Nature 424: 1057-1061

31. Xia XX, Zhi-Gang Qiana, Chang Seok Kib, Young Hwan Parkb, David LK, et al. (2010) Native-sized recombinant spider silk protein produced in metabolically engineered Escherichia coli results in a strong fiber. Proc. Natl. Acad. Sci. USA 107: $14059-14063$

32. Sampath S, Thomas Isdebski, Janelle EJ, Joel VA, Robert WH, et al. (2012) X-ray diffraction study of nanocrystalline and amorphous structure within major and minor ampullate dragline spider silks. Soft Matter 8: 6713-6722. 